\title{
Álcool e gravidez
}

MARINELA FONSECA*

\section{RESUMO}

A Organização Mundial de Saúde considera que não se deve beber rigorosamente nada se uma mulher estiver grávida ou a amamentar. A maioria das mulheres consome álcool antes de saber que está grávida. 0 consumo de álcool por uma mulher grávida pode produzir alterações físicas, cognitivas e comportamentais irreversíveis na criança que está para nascer. A mais grave dessas consequências é o Síndrome Fetal Alcoólico (SFA), cujos critérios mínimos de diagnóstico são: atraso de crescimento pré- ou pós-natal, envolvimento do sistema nervoso, atraso do desenvolvimento neuropsicomotor, alteração do Coeficiente de Inteligência e do comportamento e dismorfismo facial. Pelo menos dois dos sinais apresentados a seguir devem estar presentes: microcefalia, microflalmia e/ou fissura palpebral pequena, filtro nasal hipoplásico com lábio superior fino e hipoplasia do maxilar. Outras das consequência do consumo de álcool durante a gravidez são o aparecimento na criança que vai nascer, do Distúrbio do Desenvolvimento Neural Relacionado ao Álcool e Defeitos do Nascimento Relacionado com o Álcool. A prevenção destas doenças é feita quando a abstinência de álcool numa mulher grávida é total.

Palavras-Chave: Gravidez; Álcool; Sindrome Fetal Alcoólico; Sofrimento Fetal.
*Assistente Graduada Medicina Geral e Familiar Centro de Saúde de Cascais - Sede

Na sequência do I Curso Pós-Graduado de Abordagem do Consumo Excessivo de Álcool nos Cuidados de Saúde Primários, 03/01/2006, a FML, sensibilizou e

formou, no Centro de Saúde de Cascais, Sede e Extensão do Estoril, 28 Profissionais de Saúde, dias 13 e 26/11/06 para os Problemas Relacionados com o Consumo Excessivo de Álcool.

\section{INTRODUÇ̃̃O}

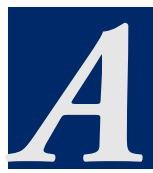

utilização de bebidas alcoólicas está fortemente enraizada na cultura dos portugueses e dos restantes europeus. A Europa continua a ser a região do mundo que mais produz e consome bebidas alcoólicas. O consumo de álcool na União Europeia está relacionado com 20 a 40\% das admissões psiquiátricas, 20 a $40 \%$ dos suicídios masculinos, 40 a $60 \%$ dos incidentes violentos e com cerca de 30\% dos acidentes de viação. $^{1}$

Causando cerca de 60 tipos de doenças e problemas (incluindo acidentes e ferimentos, cancros e doenças do coração e doenças cardiovasculares, o álcool é responsável por 7,4\% de todos os problemas de saúde e mortes prematuras da União Europeia. ${ }^{2}$

A Organização Mundial de Saúde considera que não se deve beber rigorosamente nada se uma mulher estiver grávida ou a amamentar. ${ }^{3}$ No entanto a maioria das mulheres consome álcool, antes de saber que está grávida. O uso de álcool durante a gravidez é um risco tanto para a saúde materna, quanto para a do feto porque, como o álcool atravessa a barreira placentária, a alcoolemia do feto e da mãe são idênticas. O mesmo acontece com as mulheres que amamentam. O consumo de álcool numa mulher grávida, pode produzir alterações físicas, cognitivas e comportamentais irreversiveis na criança que está para nascer. $\mathrm{O}$ aumento do consumo do álcool é responsável por um aumento da incidência de malformações, em percentagens que podem ultrapassar os 30 \% nas bebedoras excessivas, podendo as mesmas serem significativas em mulheres que consomem doses superiores a $30 \mathrm{cl}$ de álcool por semana, o que equivale a um consumo diário de 4 dl de vinho de 11 graus. ${ }^{4}$

O álcool tem efeitos tóxicos directos no processo de divisão celular, sendo o risco de malformações tanto mais elevado, quanto a alcoolização da grávida se fizer por períodos agudos e prolongados nos primeiros meses de gravidez, provocando alcoolémias elevadas durante o período embrionário. Depois do nascimento, o efeito do álcool consumido durante a gravidez, pode reflectir-se nas 12 horas após o parto, podendo desencadear no bebé um verdadeiro quadro de privação alcoólica, que se pode caracterizar por agitação, tremores, perturbações do sono, hipertonia muscular e por vezes convulsões, consecutivos à secção do cordão umbilical que mantinha a alcoolemia na circulação fetal. ${ }^{5}$ Diversos factores podem contribuir para o aparecimento de problemas no feto, tais como o padrão de consumo de álcool, metabolismo materno, susceptibilidade genética, período de gestação em que o álcool foi consumi- 


\section{DossiER}

Problemas ligados ao Álcool do e vulnerabilidade nas diferentes regiões cerebrais da criança. ${ }^{6}$

\section{Síndomes Relacionados COM O CONSUMO}

\section{Materno de Álcool na Gravidez}

A mais grave das consequências relacionadas com o consumo de álcool durante a gravidez é o Síndrome Fetal Alcoólico (SFA), descrito pela primeira vez por Jones e Smith em 1973. ${ }^{7}$ O Sindrome Fetal Alcoólico é a mais conhecida causa de atraso mental na civilização ocidental. ${ }^{8}$ Segundo a OMS, por ano, nascem cerca de 12.000 crianças com SFA. A incidência do SFA nos Estados Unidos varia de 0,5 a 3 por 1.000 nascimentos vivos na maioria das populações, no entanto com taxas muito mais elevadas em algumas comunidades. ${ }^{9}$ Alguns dos sintomas podem ser observados até que a criança complete os 3-4 anos. 4 a 10\% das crianças nascidas de mães dependentes do álcool, apresentam SFA. ${ }^{10}$ Os critérios mínimos para o diagnóstico de SFA são: ${ }^{11}$

1. Atraso de crescimento pré- ou pósnatal.

2. Envolvimento do sistema nervoso, atraso do desenvolvimento neuropsicomotor (DNPM) e alteração do Coeficiente de Inteligência e do comportamento.

3. Dismorfismo facial. Pelo menos dois dos sinais apresentados a seguir de-

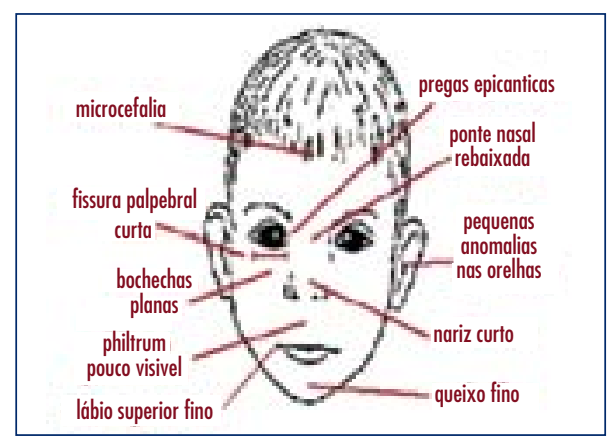

Figura 1. Alterações faciais características do SFA. Fonte: Acohol Health \&Rearch World. 1994. vem estar presentes: microcefalia, microflalmia e ou fissura palpebral pequena, filtro nasal hipoplásico com lábio superior fino e hipoplasia do maxilar (Figura 1).

Outros sinais e sintomas podem ser observados no SFA: alterações articulares, defeitos de postura dos pés, pectus excavatum, defeitos do septo ventricular, tetralogia de Fallot, estrabismo, má oclusão dentária, perdas auditivas, dentes pequenos, hipospadia, hidronefrose, hisurtismo infantil, hérnias umbilicais ou diafragmáticas. ${ }^{12}$

Os restantes $90 \%$, das crianças nascidas, filhas de mães dependentes de álcool, podem ser portadoras do Distúrbio do Desenvolvimento Neural Relacionado ao Álcool (DDNR) e Defeitos do Nascimento Relacionado ao Álcool (DNRA). As características mais graves, são os sintomas invisiveis dos danos neurológicos que resultam da exposição pré-natal ao álcool, e que podem incluir o défice de atenção, défice da memória, hiperactividade, dificuldade com conceitos abstractos, habilidades pobres de resolver os problemas, inabilidade de discernimento no uso do dinheiro, dificuldade em aprender com as consequências, comportamento social imaturo, amizade imprópria com desconhecidos, falta de controlo sobre as emoções, controlo pobre do impulso e julgamento pobre. ${ }^{13}$

Os adultos com SFA têm dificuldade em manter a sua independência bem-sucedida. Têm dificuldade em permanecer na escola, manter o emprego ou sustentar relacionamentos saudáveis.

\section{Prevencão do SFA, DDNRA E DNRA}

As patologias atrás descritas podem ser $100 \%$ prevenidas com a abstinência total de álcool, quando a mulher está grávida.

Os factores protectores contra as complicações sociais e psicológicas re- 
lacionados com o SFA incluem: relacionamentos familiares estáveis, diagnóstico, aparecimento do Síndrome antes dos 6 anos de idade, ausência de abuso sexual ou violência física, rotina estável e imune a mudanças periódicas de residência ou cidade, ausência de privações sociais e presença de acompanhamento especializado. ${ }^{14}$

\section{CONCLUSÃO}

Não há uma abordagem terapêutica para o SFA, mas sim para as suas complicações clínicas, tais como cardiopatias, convulsões, alterações oftalmológicas, dentárias, ortopédicas e psiquiátricas entre outras. A participação da família e a atenção dos factores ambienciais capazes de comprometer a adaptação desses indivíduos são estratégias importantíssimas para melhorar o prognóstico destes indivíduos. ${ }^{15}$ A ocorrência de distúrbios do comportamento é frequente entre os portadores da SFA.

Cabe principalmente ao Médico de Família a prevenção do consumo de álcool das suas utentes no período gestacional, orientando-as e alertando-as sobre a sua responsabilidade para com a saúde do seu futuro filho.

\section{REFERÊNCIAS BIBLIOGRÁFICAS}

1. Rehn N, Room R, Edwards G. Alcohol in the European Region: consumption, harm and policies. Copenhagen: WHO Regional Ofiice for Europe; 2001.

2. Rehm J, Room R, Graham K, Monteiro M ,Gmel G, Sempos CT. The relationships of average volume of alcohol consumption and patterns of drinking to burden of disease. an overview. Addiction 2003 Sep; 98 (9): 1209-28.

3. Babor T, Higgins-Biddle J. Brief intervention for hazardous and harmful drinking: a manual for use in primary care. Geneva: WHO; 2001.

4. Rosett HL, Weimer L. Alcohol and the foetus. New York: Oxford University Press; 1984.

5. Mello ML, Barrias JC, Breda JJ. Álcool e problemas ligados ao álcool. Lisboa: DirecçãoGeral de Saúde; 2001.

6. Maier SE, West JR. Drinking patterns and alcohol-related birth defects. Alcohol Res Health 2001; 25 (3): 168-74.

7 Jacobson JL, Jacobson SW. Effects of prenatal alcohol exposure on child development. Alcohol Res and Health 2002; 26 (4): 282-6.

8 National Institute on Alcohol Abuse and Alcoholism NIAA, oitava publicação no 94-3699 do relatório especial $\mathrm{NIH}$.

9 Stratton, K. Howe C, Battaglia F, editors. Fetal Alcohol Syndrome: diagnosis, epidemiology, prevention, and treatment. Washington, DC: National Academy Press; 1996.

10 May PA, Gossage JP. Estimating the prevalence of fetal alcohol syndrome: a summary. Alcohol res Health 2001; 25 (3): 159-67.

11 Sokol RJ, Clarren SK. Guidelines for use of terminology describing the impact of prenatal alcohol on the offspring. Alcohol Clin Exp Res 1989 Aug; 13 (4): 597-8.

12 Ellenhorn MJ, Schonwald S, Ordog G, Wasserberg J. Alcohols and glycols. In: Ellenhorn MJ, editor. Ellenhorn's Medical Toxicology: diagnosis and treatment of human poisoning. Baltimore, MD: Williams \& Wilkins; 1997.

13. Coles CD, Platzman KA, Raskind-Hood CL, Brown RT, Falek A, Smith IE. A comparison of children affected by prenatal alcohol exposure and attention deficit, hyperactivity disorder. Alcohol Clin Exp Res 1997 Feb; 21 (1): 150-61.

14. Streissguth AP, Barr HM, Kogan J, Brookstein FL. Final report: understanding the ocurrance of secundary disbilities in clients with fetal alcohol syndrome (FAS) and fetal alcohol effects (FAE). Seattle: University of Washington Publication Services; 1996.

15. Streissguth AP, Barr HM, Kogan J, Brookstein FL. Final report: understanding the ocurrance of secundary disbilities in clients with fetal alcohol syndrome (FAS) and fetal alcohol effects (FAE). Seattle: University of Washington Publication Services; 1996.

\section{Endereço para Correspondência} Marinela Fonseca

Parque Oceano Lote $7,2^{\circ}$ Dto

Sto Amaro Oeiras, 2780-323 Oeiras

E-mail: mduque@vizzavi.pt 


\section{DOSSIER}

Problemas ligados ao Álcool

\section{ALCOHOL AND PREGNANCY}

\section{ABSTRACT}

World Health Organization considers pregnant and suckle women shoud not drink alcohol at all. Most women drink alcohol without knowing their pregnangy. Drinking alcohol while pregnant may produce irreversible physical, cognitive and behaviour disturbances in the growing embryo. The worse disease related with alcohol abuse is known as Fetal Alcohol Syndrome characterized by: late growing before and after birth, nervous system envolvement, late psycomotor development, QI and behaviour disturbance and facial dismorphysm. At least two of the physical signs must be present as microcephaly, microphtalmy, small palpebral fissure, hypoplasy of nasal sept with thin superior lip and maxillary hypoplasy. Other consequences of alcohol drinking during pregnancy are the Alcohol Realted Neurodevelopmental Disorder and the Alcohol Related Birth Defects. The prevention of these diseases is obtained with total abstinence of alcohol drinking while pregnant.

Key-Words: Pregnancy; Alcohol; Alcohol Fetal Syndrome. 